\title{
Magnetic Behavior of an Iron Gluconate/Polyaniline Composite
}

\author{
Yusuke Koshikawa and Hiromasa Goto * (D) \\ Department of Materials Science, Faculty of Pure and Applied Sciences, University of Tsukuba, \\ Tsukuba 3058573, Japan; s2120329@s.tsukuba.ac.jp \\ * Correspondence: gotoh@ims.tsukuba.ac.jp
}

Citation: Koshikawa, Y.; Goto, H. Magnetic Behavior of an Iron Gluconate/Polyaniline Composite. J. Compos. Sci. 2021, 5, 252. https:// doi.org/10.3390/jcs5090252

Academic Editor: Konda Gokuldoss Prashanth

Received: 14 August 2021 Accepted: 15 September 2021 Published: 19 September 2021

Publisher's Note: MDPI stays neutral with regard to jurisdictional claims in published maps and institutional affiliations.

Copyright: (c) 2021 by the authors. Licensee MDPI, Basel, Switzerland. This article is an open access article distributed under the terms and conditions of the Creative Commons Attribution (CC BY) license (https:// creativecommons.org/licenses/by/ $4.0 /)$.
Abstract: Oxidative polymerization conducted the synthesis of polyaniline in the presence of iron gluconate in the water. Iron gluconate is present in the resulting polyaniline (PANI). The PANI composite exhibited multiple signals in electron spin resonance, including half-field resonance of multiple spin states, the center-field resonance of polarons as radical cations in conducting polymer, and a signal from a defect in the main chain. Infrared (IR) absorption spectroscopy measurements confirmed the chemical structure of the PANI composite. The composite exhibits the mixed magnetism of PANI as a conducting polymer and Fe ions in the composite according to superconducting interference device (SQUID) measurements. Combining organic-conjugated polymers and inorganic materials can result in a unique magnetism.

Keywords: conductive polymer; polyaniline; magnetism; iron gluconate

\section{Introduction}

Polymer magnetism has been studied since 1980 to synthesize organic magnets [1-3]. Oxy-radicals have been mainly used as the spin species for magnetic polymers. A variety of organic magnetic materials have been synthesized via $\pi$-electron frameworks. For example, poly(para-phenylenevinylene) derivatives bearing oxyradicals that exhibit ferromagnetic behavior at low temperatures have been synthesized. The magnetic polymers, on the other hand, are generally unstable in ambient air. As a result, the metal-free magnetic polymer's instability is a disadvantage in applications. Recently, polymers with magnetism were investigated using a combination of inorganic materials [4-8]. The combination of inorganic materials and conducting polymers is used in this study to create air-stable magnetic-electrical conducting plastics. Magneto-active materials with chemical-physical functionalities have recently been developed [9-11].

Further, the composite formation can expect magnetic interaction between inorganic magnetic materials and organic conducting polymers. We employ iron gluconate (IG) as a chiral filler to polyaniline. The composite is prepared in the polymerization process of aniline (monomer) in the presence of IG as a convenient procedure. Recently, chiral conjugated polymers have been developed [12-15]. Composite magnetic polymer with chiral filler can produce magneto-optically active polymers. Our research goal on the polyaniline/optically active compound composite is a preparation of magneto-optically active soft devices.

Aniline was polymerized with ammonium persulfate in the presence of IG to produce a polymer of the polyaniline (PANI) composite with magnetism in this study. The resulting composite was subjected to infrared absorption (IR) and UV-visible optical absorption spectroscopy. Furthermore, the magnetic properties of the composite were assessed using electron spin resonance (ESR) and a superconducting quantum interference device (SQUID).

\section{Synthesis and Characterization Method}

The synthesis of polyaniline-based composite in the presence of IG was carried out for obtaining the magnetic composite (Scheme 1). First, in a round bottom flask with a $1 \mathrm{~cm}$ 
stirring bar, IG (2.004 g), aniline (monomer, $0.504 \mathrm{~g}$ ), and sulfuric acid ( $0.512 \mathrm{~g})$ were mixed in $100 \mathrm{~mL}$ of distilled water. Then, $2.502 \mathrm{~g}$ of ammonium persulfate was added and stirred at approximately $0{ }^{\circ} \mathrm{C}$. After $24 \mathrm{~h}$, the reaction mixture was filtered and washed with a large volume of water. The solution was filtered. The product was washed with a large volume of methanol. Then, the solution was filtered again. The final product was dried under reduced pressure to yield $0.339 \mathrm{~g}$ of a blue-violet powder labeled as IG-PANI.
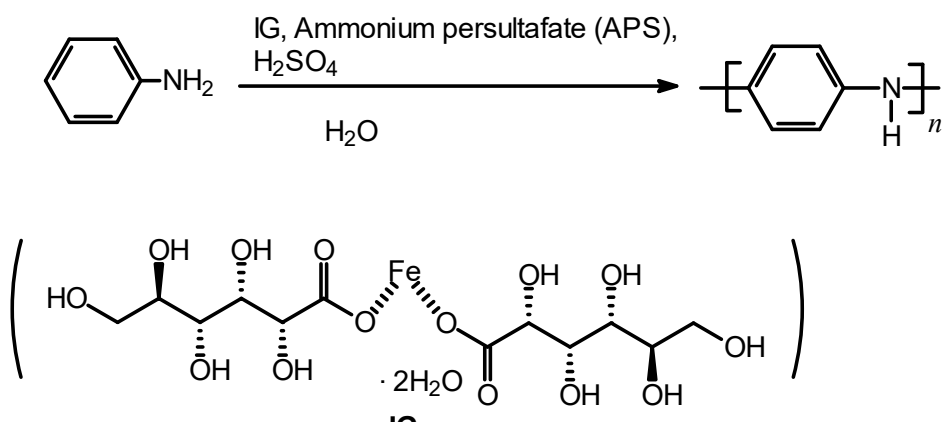

IG

Scheme 1. Synthesis for polyaniline-iron gluconate composite (labeled IG-PANI). Molecular structure of IG.

For Fourier transform infrared absorption spectroscopy measurements with an FT-IR 4600 , the $\mathrm{KBr}$ method was used (JASCO, Tokyo). The ESR of a solid sample packed into a 5-mm quartz tube was measured using an X-band JEOL JES TE-200 spectrometer. The magnetic susceptibility of the polymer was measured using a superconductor interference device (SQUID) (Quantum Design CA, Magnetic property measurement system, MPMS).

\section{Results and Discussion}

The ultraviolet-visible (UV-vis) absorption spectra of IG-PANI dissolved in Nmethylpyrroridone (NMP) are shown in Figure 1. Absorption due to the $\pi-\pi^{*}$ transition of the main chain was observed at $354 \mathrm{~nm}$. At $631 \mathrm{~nm}$, there was broad absorption due to the doping band consisting of benzenoid and quinonoid structures [16-18]. The absorption band of IG-PANI is $9 \mathrm{~nm}$ blue-shifted from that of pure PANI ( $\lambda_{\max \text { (polarons) }}: 640 \mathrm{~nm}$ ), indicating IG made distortion of the main chain of PANI to decrease effective conjugation length. No distinct absorption from ion ions (i.e., $d-d$ transition) was observed at this range.

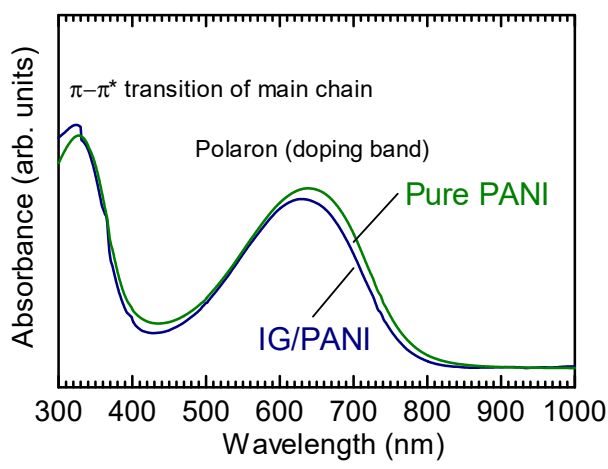

Figure 1. Ultraviolet-visible absorption spectra of pure PANI and IG-PANI.

The polarons in the main chain of PANI were generated by sulfuric acid, which was used as a dopant during the polymerization reaction.

Infrared absorption (IR) measurement with the $\mathrm{KBr}$ method for the composite was carried out to confirm the chemical structure. Figure 2 displays the IR results for IG, pure PANI, and IG-PANI. Absorption bands for IG at $1621 \mathrm{~cm}^{-1}$ and $1400 \mathrm{~cm}^{-1}$ are due to asymmetric $\mathrm{COO}^{-}$stretching and symmetric $\mathrm{COO}^{-}$stretching [19]. An absorption at 
$1086 \mathrm{~cm}^{-1}$ is assignable to $\mathrm{C}-\mathrm{O}$ deformation $\left(\gamma_{\mathrm{C}-\mathrm{O}}\right)$. Absorption bands due to quinonoid (Q) and benzenoid (B) structures were observed for pure PANI and IG-PANI. Further, the sequence of Q-B-Q and B-B-B in the main chain was confirmed in the IR [20]. Absorption due to $\mathrm{S}=\mathrm{O}$ and in-plane bending vibration $(\delta)$ of $\mathrm{C}-\mathrm{H}$ was observed at a low wavelength range. IG-PANI showed basically the same IR absorptions as pure PANI except for the complicated signals at $1000-1200 \mathrm{~cm}^{-1}$, which contained absorptions due to $\mathrm{OH}$ moiety. The IR results suggest that IG-PANI basically shows absorptions of PANI with quite a small amount of iron gluconate. However, an absorption related to the iron atom was not observed in this range.

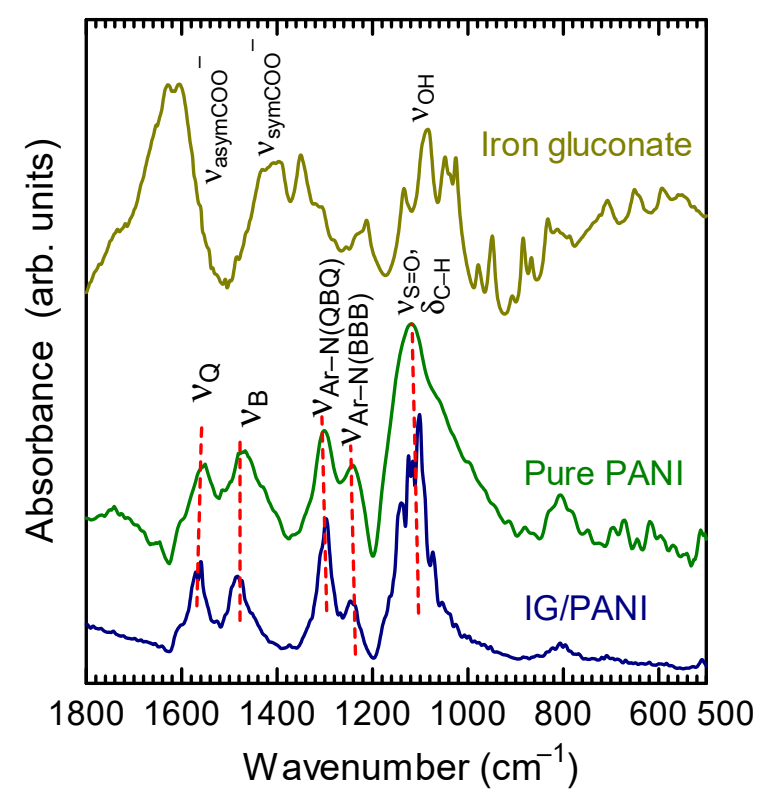

Figure 2. IR absorption spectrum of IG-PANI.

The ESR measurement results for IG-PANI in the form of a solid powder at room temperature (ca. $25^{\circ} \mathrm{C}$ ) are shown in Figure 3a-c. Iron resonance, structural defects, polyaniline, and iron ion paramagnetic resonance are all present in the signal. The resonance in the center field at $\mathrm{g}=2.001$ (Figure 3a) could be caused by a paramagnetic species' lattice defect (defect in the main chain). This type of ESR signal was observed for $\mathrm{Rb}_{3} \mathrm{C}_{60}$, except for the half-field resonance in the current sample. The ESR center signal of polarons as charge carriers (radical cations) overlapped with this signal. The linewidth of the center-field signal $\left(\Delta H_{\mathrm{pp}}\right)$ is $81.9 \mathrm{mT}$. In addition, a signal from multiple spins was observed in the half-field range at $\mathrm{g}=4.227$ with a linewidth of $20.7 \mathrm{mT}$, as shown in Figure $3 \mathrm{a}, \mathrm{b}$ (magnification). This can be the triplet state of spins in the composite. Figure $3 \mathrm{c}$ shows a magnification of the lattice defect signal $\left(\mathrm{g}=2.001, \Delta H_{\mathrm{pp}}=2.2 \mathrm{mT}\right)$. Figure $3 \mathrm{~d}$ displays the ESR signal for pure PANI prepared with no IG, showing a narrow $\Delta H_{\mathrm{pp}}$ value at $\mathrm{g}=2.004\left(\Delta H_{\mathrm{pp}}=0.8 \mathrm{mT}\right)$ and no signal at $\mathrm{g}=4$ (half-field resonance). The comparison of IG-PANI with pure PANI in the ESR demonstrated that the IG-PANI has completely different magnetic species against the pure PANI. Figure $3 e$ depicts the g-value as a function of temperature. Further, Figure $3 \mathrm{f}$ displays a change in the ESR intensity and $\Delta H_{\mathrm{pp}}$ of the IG-PANI composite at $\mathrm{g}=2.001$. The g-value was constant from high temperature to low temperature with cooling, indicating charge species of the composite was no change. While the signal intensity was increased with the decrease of temperature, the line width was constant. 

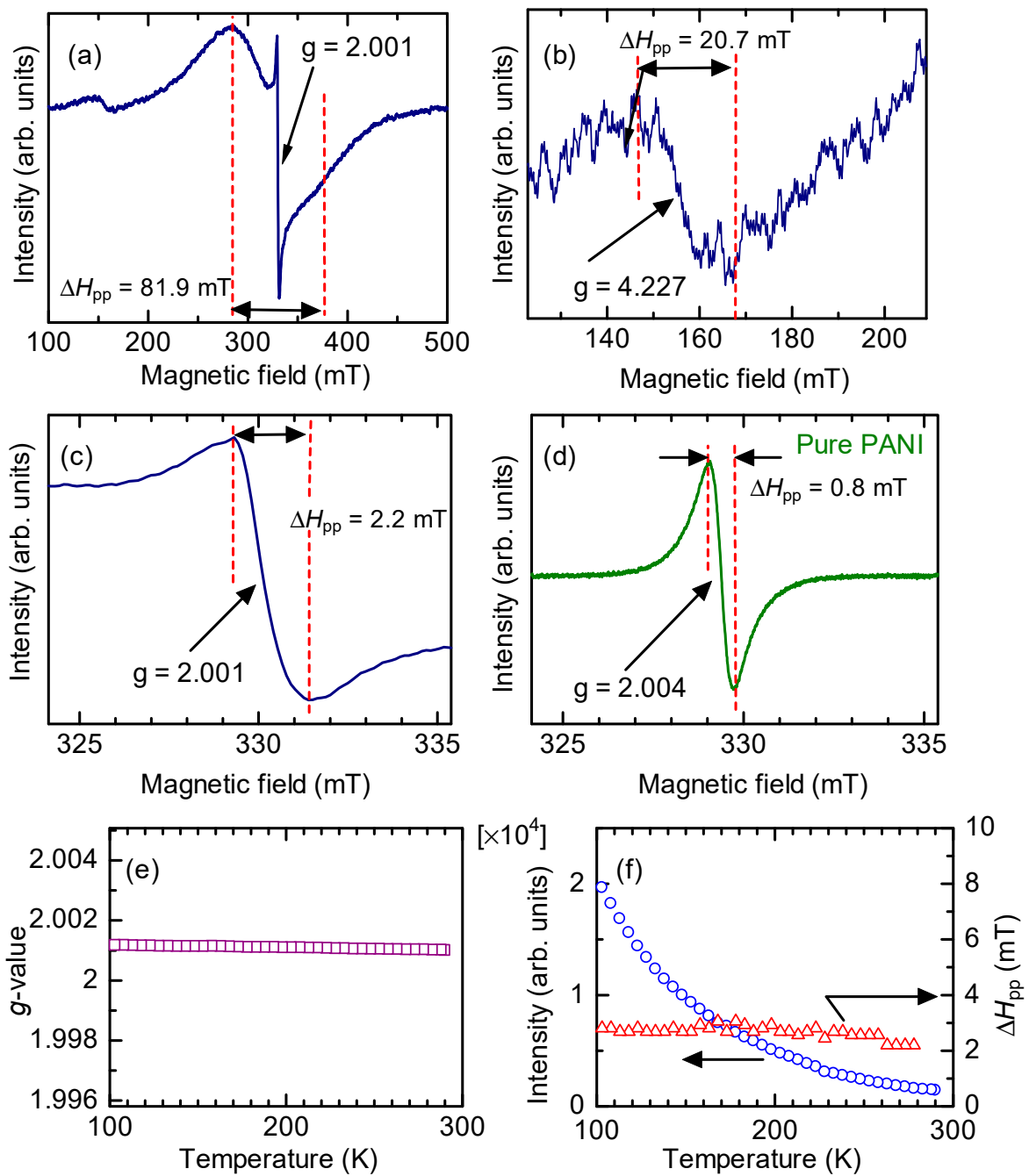

Figure 3. (a) Electron spin resonance (ESR) of IG-PANI at 100-500 mT range. (b) Half-field resonance. (c) ESR signal at around $\mathrm{g}=2$ as the center-field resonance. (d) ESR signal of pure PANI. (e) g-values vs. temperature. (f) Peak-to-peak peak line width $\left(\Delta H_{\mathrm{pp}}\right)$ of IG-PANI as a function of temperature.

Magnetic measurements using the SQUID reveal the entire magnetic behavior of the sample.Figure 4a shows $\chi$ vs. $T$ plots $(\chi=$ magnetic susceptibility). The $\chi$ value of the composite was increased with temperature. $1 / \chi$ as a function of $T$ shows an inflection point at ca. $270 \mathrm{~K}$, implying the occurrence of a magnetic phase transition (Figure 4b). An extrapolation at the high-temperature range directs to the zero value, implying ferromagnetic behavior. $\chi T$ vs. $T$ as Currie plots indicate that the sample showed a maximum of $\chi T$ at $262 \mathrm{~K}$ and gradually decreased (Figure $4 \mathrm{c}$ ) to a low-temperature range. Such behavior is usually attributed to zero-field splitting or intermolecular antiferromagnetic coupling [21,22]. This composite may contain ferromagnetic and antiferromagnetic components. $\chi \mathrm{T}$ vs. $1 / \mathrm{T}$ plots as Ising plots demonstrate a magnetism change (Figure $4 \mathrm{~d}$ ). In the previous study, the SQUID measurement results for pure PANI and PANI-poly(styrenesulfonic acid) indicated that the polymer shows Pauli's paramagnetism. However, such an inflection point in the $x$ vs. $T$ plots at the high-temperature range was not observable $[23,24]$. Therefore, the unique magnetic behavior of IG-PANI was derived from the interaction of IG and PANI in the composite form. 

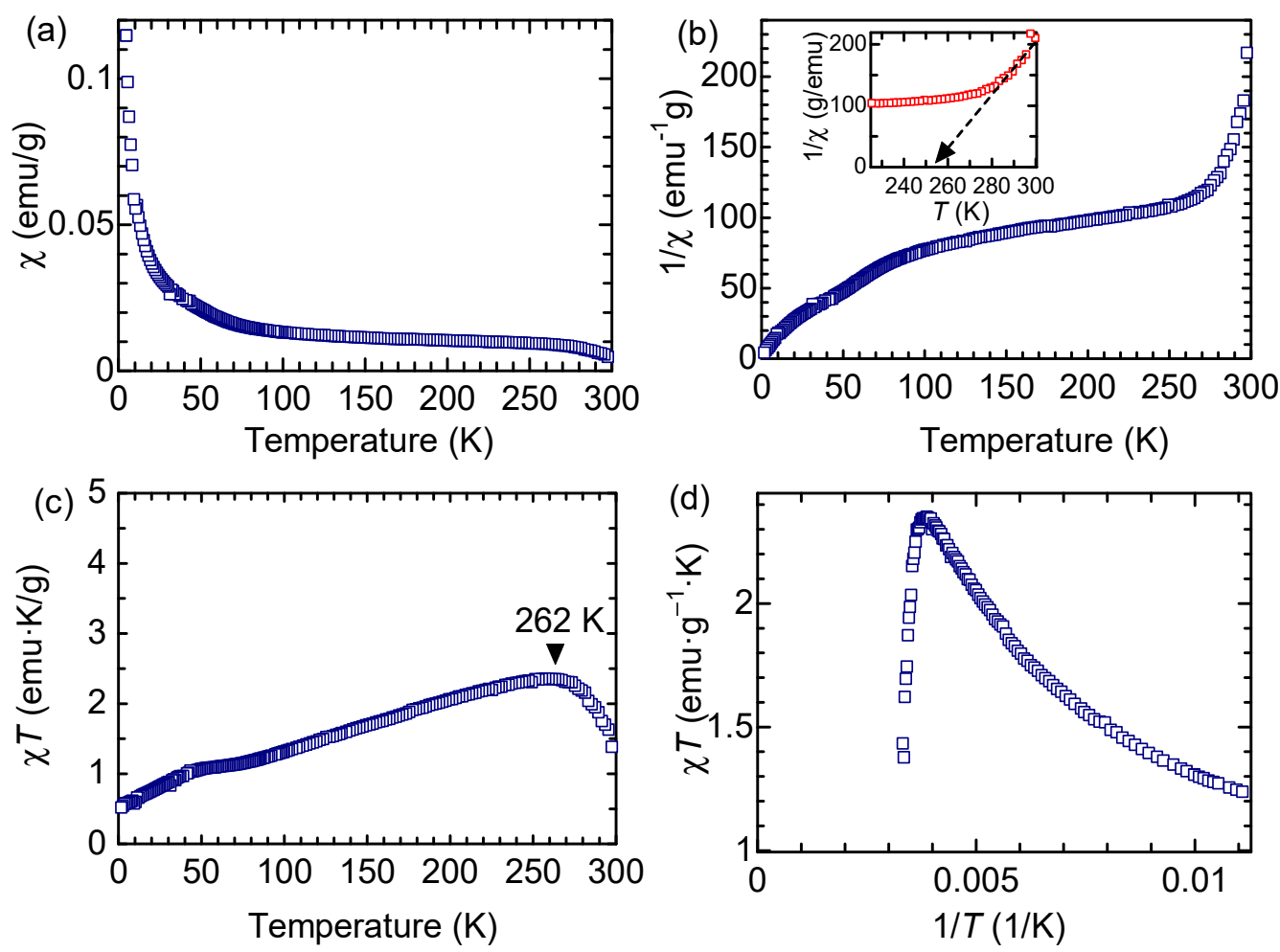

Figure 4. (a) $\chi$ vs. $T$ plots. (b) $1 / \chi$ vs. $T$ plots. (c) $\chi T$ vs. $T$ as Currie plots. (d) $\chi T$ vs. $1 / T$ plots as Ising plots. The inset of (b) shows a magnification of $1 / \chi$ vs. $T$ plot.

The sample's magnetism is complicated due to the mixed magnetism from the composite's polarons, iron ions, and lattice defects. In addition, external stimuli, such as light irradiation, may cause magnetism to be tuned for the magnetic composite. The combination of magnetism in organic-inorganic material composites can result in magnetic materials that are previously unseen. It is expected that the composite will be processable for producing thin films, sheets, and textiles.

Author Contributions: Y.K. synthesized the polymer and carried out the SQUID and ESR measurements. H.G. carried out the ESR measurements and conducted this project. All authors have read and agreed to the published version of the manuscript.

Funding: This research was supported by the Japan Society for the Promotion of Science, Grants-inAid for Scientific Research (No. 20K05626).

Institutional Review Board Statement: Not applicable.

Informed Consent Statement: Not applicable.

Acknowledgments: We would like to thank the Open Facility, Research Facility Center for Science and Technology, and University of Tsukuba.

Conflicts of Interest: The authors declare no conflict of interest.

\section{References}

1. Iwamura, H.; Murata, S. Magnetic Coupling of Two Triplet Phenylnitrene Units Joined Through an Acetylenic or a Di-Acetylenic Linkage. Mol. Cryst. Liq. Cryst. Incorp. Nonlinear Opt. 1989, 176, 33-47. [CrossRef]

2. Iwamura, H. Design and Demonstration of Ferromagnetic Exchange Interactions in Organic Molecules. Mol. Cryst. Liq. Cryst. Sci. Technol. A Mol. Cryst. Liq. Cryst. 1993, 232, 233-250. [CrossRef]

3. Nishide, H.; Ozawa, T.; Miyasaka, M.; Tsuchida, E. A Nanometer-Sized High-Spin Polyradical: Poly(4-phenoxyl-1,2phenylenevinylene) Planarily Extended in a Non-Kekulé Fashion and Its Magnetic Force Microscopic Images. J. Am. Chem. Soc. 2001, 123, 5942-5946. [CrossRef] 
4. Hong, K.; Choe, M.; Kim, S.; Lee, H.-M.; Kim, B.-J.; Park, S. An Ultrastretchable Electrical Switch Fiber with a Magnetic Liquid Metal Core for Remote Magnetic Actuation. Polymers 2021, 13, 2407. [CrossRef] [PubMed]

5. Park, S.; Oh, Y.; Jung, D.; Lee, S.H. Effect of Cellulose Solvents on the Characteristics of Cellulose/Fe2O3 Hydrogel Microspheres as Enzyme Supports. Polymers 2020, 12, 1869. [CrossRef] [PubMed]

6. Wei, D.-H.; Chang, J.-H.; Hsu, C.-C.; Yang, C.-J.; Liang, Y.-C.; Dong, C.-L.; Yao, Y.-D. Controlled Magnetic Isolation and Decoupling of Perpendicular FePt Films by Capping Ultrathin $\mathrm{Cu}(002)$ Nano-Islands. J. Compos. Sci. 2021, 5, 140. [CrossRef]

7. Nacy, S.; Youssef, G. Demagnetization Effect on the Magnetoelectric Response of Composite Multiferroic Cylinders. J. Compos. Sci. 2021, 5, 139. [CrossRef]

8. Jouyandeh, M.; Ganjali, M.R.; Karami, Z.; Rezapour, M.; Bagheri, B.; Zarrintaj, P.; Mouradzadegun, A.; Habibzadeh, S.; Saeb, M.R. Thermal Analysis of Crosslinking Reactions in Epoxy Nanocomposites Containing Polyvinyl Chloride (PVC)-Functionalized Nickel-Doped Nano-Fe3O4. J. Compos. Sci. 2020, 4, 107. [CrossRef]

9. Ramos, P.; Amorin, H.; Ricote, J.; Castro, A.; Alguero, M. Insights into the Performance of Magnetoelectric Ceramic Layered Composites. J. Compos. Sci. 2017, 1, 14. [CrossRef]

10. Correa, A.A.; Pereira, E.C.; de Oliveira, A.J.A. Magnetic Properties of Conducting Polymers. In Emerging Research in Science and Engineering Based on Advanced Experimental and Computational Strategies. Engineering Materials; La Porta, F., Taft, C., Eds.; Springer: Cham, Germany, 2020. [CrossRef]

11. Long, Y.; Chen, Z.; Shen, J.; Zhang, Z.; Zhang, L.; Xiao, H.; Wan, M.; Duvail, J.L. Magnetic Properties of Conducting Polymer Nanostructures. J. Phys. Chem. B. 2006, 110, 23228-23233. [CrossRef]

12. Majidi, M.R.; Kane-Maguire, L.A.P.; Wallace, G.G. Facile synthesis of optically active polyaniline and polytoluidine. Polymer 1996, 37, 359-362. [CrossRef]

13. Jia, L.; Wang, C.; Zhang, Y.; Yang, L.; Yan, Y. Efficient Spin Selectivity in Self-Assembled Superhelical Conducting Polymer Microfibers. ACS Nano 2020, 14, 6607-6615. [CrossRef] [PubMed]

14. Li, X.; Yu, L.; Yu, L.; Dong, Y.; Gao, Q.; Yang, Q.; Yang, W.; Zhu, Y.; Fu, Y. Chiral Polyaniline with Superhelical Structures for Enhancement in Microwave Absorption. Chem. Eng. J. 2018, 352, 745-755. [CrossRef]

15. Mishra, S.; Kumar, A.; Venkatesan, M.; Pigani, L.; Pasquali, L.; Fontanesi, C. Exchange Interactions Drive Supramolecular Chiral Induction in Polyaniline Suryakant. Small Methods 2020, 4, 2000617. [CrossRef]

16. MacDiarmid, A.G.; Chiang, J.C.; Richter, A.F.; Somasiri, N.L.D.; Epstein, A.J. Polyaniline: Synthesis and Characterization of the Emeraldine Oxidation State by Elemental Analysis. In Conducting Polymers; Alcácer, L., Ed.; Springer: Berlin/Heidelberg, Germany, 1987; pp. 105-120. [CrossRef]

17. Nekrasov, A.A.; Ivanov, V.F.; Vannikov, A.V. Analysis of the Structure of Polyaniline Absorption Spectra Based on Spectroelectrochemical Data. J. Electroanal. Chem. 2000, 482, 11-17. [CrossRef]

18. Sindhimeshram, D.C.; Gupta, M.C. Transport properties of substituted derivatives of polyaniline. Indian J. Chem. 1995, 34, 260-277.

19. Nikolić, V.D.; Ilić, D.P.; Nikolić, L.B.; Stanojević, L.P.; Cakić, M.D.; Tačić, A.D.; Ilić-Stojanović, S.S. The synthesis and characterization of iron(II) gluconate. Savrem. Tehnol. 2014, 3, 16-24. [CrossRef]

20. Tang, J.; Jing, X.; Wang, B.; Wang, F. Infrared spectra of soluble polyaniline Synth. Met. 1988, 24, $231-238$.

21. Yang, E.-C.; Chang, Y.-Y.; Huang, S.-Y.; Hong, L.-X.; Lee, G.-H.; Sheu, H.-S.; Chang, C.-K. Novel Structures and Magnetic Properties of Two [Mn2] Complexes with 2,4-di-2-pyridyl-2,4-pentanediol as the Ligand. Magnetochemistry 2019, 5, 43. [CrossRef]

22. Yang, X.; Xu, Q. Bimetallic Metal-Organic Frameworks for Gas Storage and Separation. Cryst. Growth Des. 2017, 17, 1450-1455. [CrossRef]

23. Kahol, P.K.; Ho, J.C.; Chen, Y.Y.; Wang, C.R.; Neeleshwar, S.; Tsai, C.B.; Wessling, B. On Metallic Characteristics in Some Conducting Polymers. Synth. Met. 2005, 151, 65-72. [CrossRef]

24. Djurado, D.; Pron, A.; Travers, J.-P.; Duque, J.G.S.; Pagliuso, P.G.; Rettori, C.; Chinaglia, D.L.; Walmsley, L. Magnetic Field Dependent Magnetization of a Conducting Plasticized Poly(aniline) film. J. Phys. Condens. Matter 2008, 20, 285228. [CrossRef] 